\title{
Surmounting the Cartesian Cut: Klein Bottle Logophysics, the Dirac Algebra and the Genetic Code
}

\begin{abstract}
Diego Lucio Rapoport
Abstract

We reintroduce the Klein Bottle (KB) logophysics at the foundations of the unification of quantum geometry, cell biology, embryology and evolution, to extend it to the genetic code and allosteric recognition. We find that the genetic code has three possible fractal topologies: two different families of KBs embedded in HyperKBs, or still a 2-torus, depending which pair of three subalphabets for the genetic "letters" is chosen; this does not require the double helix. We complete the genetic codification of embryological differentiation introduced in an accompanying article. We discuss the Hadamard-KB representation of this codification and discuss its robustness and that of embryological development with respect to environmental noise. We discuss this KB codification of the 64 codons with its isomorphic representation in terms of the Dirac Algebra (DA) of Quantum Mechanics, in the Nilpotent Universal Computational Rewrite System (NUCRS), a self-referential syntax that generates much of mathematics and the fundamental symmetries of physics. We show that the double helix can be obtained from the duplication of the KB associated with the bistable perception of the tetrahedron representation of the four genetic letters. The DA and its double tetrahedron codification in NUCRS is found to be associated with a pair of superposed KBs, yielding the double 3D (space and momentum) space of physics, and the double helix of DNA, which thus appears related to a pair of KBs, being the case that only one is necessary to construct the genome. We show that the depth-time variable of visual perception materializes as space Thus, the DA is derivable from two KBs, and the purported dualism of physics as presented in NUCRS, is rendered to be based in the non-dualistic KBL. We discuss the relations with a theory of evolutions, and between biological periodicities and the KB of the Mendeleev table (Boeyens). We present relations between metacognition and the HyperKlein Bottle, and evolution in terms of time waves defining in toto the different structures. We present rudiments of the ontoepistemology of the Hyper KB Logic, its relations with the anthropic principles and the universal physical constants, which are found to be contextual.
\end{abstract}

Key Words: klein bottle logic, time waves, genetic code, allosterics, logophysics

NeuroQuantology 2011; 4: 862-881

\section{Introduction}

A logophysical theory surmounting the conception that separates the objective and subjective realms (Cartesian Cut), based

Corresponding author: Diego Lucio Rapoport

Address: Departamento de Ciencia y Tecnologia, Universidad Nacional de Quilmes, Bernal, Buenos Aires, Argentina.

Phone: +54-11-4555-6275

e-mail: diego.rapoport@gmail.com

Received November 25, 2011. Revised December 10, 2011.

Accepted December 12, 2011. upon Klein Bottle logic (KBL) and torsion fields was proposed in (Rapoport 2011a, b), and applied to cell biology and embryology in the accompanying article (Rapoport, 2011c).

The objective of this article is to extend this conception to genetics, its relations to Quantum Mechanics and a fortiori, with embryological development and evolution, and to show that the origin of 
the double helix and the double $3 \mathrm{D}$ space (coordinates) and momentum representation of physics, and of the dualism at large claimed by theoretical physics, stems instead of the purported dualistic basis, from the non-dual KBL.

With respect to the genome, we shall present several codifications of the genetic code (GC) and the self-referential topologies of it, to further apply it embryological differentiation, and to relate it with the Nilpotent Universal Computational Rewrite System (NUCRS) by (Hill and Rowlands, 2008; Rowlands, 2008). This theory is important since it produces from a selfreferential syntax, much of mathematics and physics fundamental symmetries, in terms of a codification of them that shows that NUCRS has for its mathematical backbone the Dirac Algebra of Quantum Mechanics. In doing this it shows how space, momentum, the fundamental interactions, holography, etc. are all derived from the self-referential syntax.

We shall present a simple derivation of the genetic code from the KBL, to further related the fundamental 64 codons to the Dirac Algebra of Quantum Mechanics. In particular, we shall show that the origin of the double helix and the double $3 \mathrm{D}$ space (coordinates) and momentum representation of physics, and of the dualism at large claimed by theoretical physics and biology, stems instead of the purported dualistic basis, from the non-dual KBL.

\section{Non-orientable self-referential topologies and biochemistry}

In (Rapoport, 2011c), a crucial role was ascribed to topological changes in embryological development, and extended to chemistry and cell biology (Rapoport, to appear). The non-orientable Moebius band (MB) and the Klein Bottle (KB) (from now on we may not explicit a distinction between the surface and the logic as they are fused, as we shall see next), acting in the cell's membrane adhesion sites for integrines molecules that unify the cell's interior and exterior, may be related to the logophysical onset of embryonic wave differentiation by changing the topology of the membrane to nonorientable. These are crucial molecules for the self-organization and self-regulation of the cell through its quantum tensegrity structure that extends outside of the cell to the extracellular matrix, conforming thus an holonomic living matrix, in which quantumtopological superposition and holography are at the roots of cell biology (Rapoport 2011c). The living matrix (cytoskeleton, membrane, extracelular matrix extending through the connective tissues to the body sustaining its integration) appears to act as a perceptual self-transforming system, in relation with the environment. Topological transformations of molecules and the cell's living matrix, are conceptually similar to the allosteric effect, which is regulatory, and is transmitted over a distance (alike entanglement, either topological or quantum, which in the KBL are related (Rapoport, 2011a,b; Stern, 2001) within a protein, by which the binding of an effector in one site will change the catalytic behaviour of an enzyme or the binding affinity of a binding protein in a different part of the biomolecule.

Conformational changes, for example in enzyme-substrate, antibody antigen, proteinprotein complexes, of protein to DNA, are essential to the mutual recognition of biomolecules. These adaptable stereochemical modifications point out to the biological importance of topological changes (Rapoport, to appear \& 2011c). This is analogous to the link existing between the topological representation of a given network (its wiring diagram, corresponding to the crystal structure of the protein) and the dynamics of the network itself that are supported (but not barely coincident) with its topology (Daily and Gray, 2009), similarly to the dynamical tensegrity structure on which the quantum wave propagation produces embryological development (Rapoport, 2011c).

The classic example is haemoglobin where binding of oxygen to one of the four subunits arranged at the vertices of a tetrahedron around an axis of two-fold symmetry (alike the DNA single strand of the NUCRS), increases the affinity of the others; remarkably, hemoglobin undergoes a crucial topological transformations that allows it to phagocyte.

Thus, Outside is turned Inside as is the case of the living matrix and the Klein Bottle (KB), and embryological development, for which also turning Inside-Outside is the case (Rapoport, 2011c). Thus, the Moebius Band 
(MB) and KB molecular topologies, due to their topological superpositions and through quantum field (particularly nilpotent) logical operators (Rapoport 2010a,b), can explain the origin of these distant correlations by establishing a logophysical recognition.

As is already the case of enzymes in the key and lock model proposed first by Emil Fischer, in which there is a perfect conformational complementarity, the matching is not an artefact of the parts (the emergentists' cliché, "the whole is more than the sum of its parts") but the KBL holonomy of their interaction.

A dynamical modification (the inducedfit, $I F)$ to the lock and key model appeared (Koshland, 1958): Since enzymes are flexible structures, the active site is continually reshaped by interactions with the substrate as the substrate interacts with the enzyme, alike to the behaviour of the cell's membrane with respect to the cytoskeleton and the extracelular matrix as discussed in our accompanying article to the present one.

Indeed, while the IF was based upon geometrical and topological related (orientability) issues, the discovery of natively unfolded proteins, i.e. protein systems that perform their physiological role without the need of acquiring a well defined 3D structure (Koshland, 1958), lead to enlarge the idea of 'structure' from a specific geometrical pattern to a dynamical non unique configuration (Daily and Gray, 2009). Yet, the discovery of natively unfolded proteins (Uverski, 2002), i.e., protein systems that perform their physiological role without the need of acquiring a well defined $3 \mathrm{D}$ structure, pushed the scientists to enlarge the general idea of 'structure' from a specific geometrical pattern to a dynamical system moving around a quasiattractor constituted by a not unique configuration.

Thus, we suggest that the determination of the final fit is produced in terms of the elastodynamics-quantum tensegrity which determines the overall dynamics and topology of the cell (Rapoport, 2011c). In this principle, it is crucial that molecules in an environment can transform its topologies to non-orientable, even without breaking chemical bonds (Sokolov, 1973; Herges, 2006).
We note that the conventional take on protein structure, was built upon $x$-rays crystallography, alike the origin of the double helix of genetics; yet it was remarked by (Rich, 1995) that as a method to assess the topology already of DNA, it does not have the necessary degree of resolution, which requires atomic resolution and through which the double helix was claimed to have been resolved in two cases, yet showing considerable stacking. Also, generally, the experimental verification of protein structures is impeded by the actual observation, a future that also is common to molecules at large; indeed, the very observation may change the conformation which is sensible to electromagnetic sources which are in fact determined already by their interaction with them (Boeyens, 2005).

We propose that electromagnetic resonance is at the basis of distant communication, and the guidance is informed by the quantum potential (which is also related to the torsion quantum geometry (Rapoport, 2010a) in the holistic conception of causal Quantum Mechanics by Bohm, and final shaping proceeds by topological transformations (as is the case of hemoglobin) that may involve a obliteration of the dualistic separation between Exterior and interior.

In fact, as was proposed by (Boeyens, 2005) that only a really quantum mechanical theory of molecules and chemical reactions can be realized in terms of the quantum potential in which the environment plays a crucial role in actually shaping molecules, by the absorption or emission of electromagnettic radiation from and to the environment. Thus, in this absorption and emission of electromagnetic quanta, that molecules interact with the environment and interact between themselves, and shape determinations occur (Boeyens, 2005), and in particular topological transformations changing the orientability of molecules: orientable topologies clearly indicate an Outside-Inside divide, while nonorientability means that they are intertransformed, as is the case of the $\mathrm{KB}$. The latter is the case of embryological determination (Rapoport, 2011c). We shall see below that the genetic code has both type of topologies. 
Needless to say, this conception diverges from the present take on chemical reactions, in which no holistics are considered, the environment being neglected. The usual theory, i.e., Quantum Chemistry appears not to be based on Quantum Mechanics, and it does not incorporates the environment, rather thinking of chemical reactions as occurring in closed systems (Boeyens, 2005). Furthermore, (scalar) energy landscapes are not the case in chemical reactions although the usual take assume them to be their most basic mechanism, but (vector) gradients of the imaginary component of the wave function are crucial, instead.

This absorption or emission of quanta acts on the wave function of the factorized elements (of the overall quantum function) intervening on the chemical reaction which thus become self-signified, producing a change in the imaginary components of the wave functions, which play the role of the coefficients of time operators (Rapoport, 2011c); in particular, this is the case of chemical catalytic cycles, that define the shaping of molecules by the principle of quenching of angular momentum (Boeyens, 2005). This angular momentum (neglected in Quantum Chemistry) is derived from the imaginary phase of the Schroedinger function, and by a quenching mechanism of angular momentum determines the stereochemical shape. In the ground state where the phase imaginary component of the wave function is null, no such phenomenon exists, so that as expressed before, excited states by quanta absortion or emission, which do have an imaginary phase and thus have an angular momentum gradient, are the basis for this shape determination. Of primary relevance, is the shape of the electric charge density of the molecule which does not arise from the usual hybridization schemes that purport to produce the geometry of atoms and molecules. (Boeyens, 2005). This density can invaginate due to topological transformations that occur due to molecular recognition (Sokolov, 1973), and that also has a correlate as an elastodynamical transformation, whose wavefront is identical to those of the quantum substratum as a light tensegrity structure (Rapoport, 2011c). Thus, this quantum dynamics does also manifest as an elastodynamics, the latter being the physical field which in the usual take is associated to allosterics.

So, to resume, these adaptable stereochemical modifications point out to the importance of topology -yet associated to a torsion geometry-both defined by the wave function (Rapoport, 2007), and secondly and most important, that these topologies may be changing themselves (Giuliani, 2009). The non-orientable $\mathrm{MB}$ and $\mathrm{KB}$ topologies of stereochemistry has been claimed to yield a new paradigm to organic chemistry (Sokolov, 2009; Herges, 2006; Bonchev and Rouvray, 2000), and in (Rapoport, 2011c) it was argued it plays a core role in cell biology and embryology, having them all the same organizing principle.

In this new paradigm, we contested the dualistic approach implicit to the rigid cathegorization of cell biology and the phenotypical transformations as linked to an outside and inside ontology, which permeates biology. Indeed, the $\mathrm{KB}$ has no fixed separations between inside and environment, due to its self-containment. As a result, the substrate does not simply bind to a rigid active site; the aminoacid side chains which make up the active site are molded into the precise positions that enable the enzyme to perform its catalytic function. In some cases, such as glycosidases, the substrate molecule also changes shape slightly as it enters the active site. The active site continues to change until the substrate is completely bound, at which point the final shape and charge is determined. Induced fit may enhance the fidelity of molecular recognition in the presence of competition and noise via the conformational proofreading mechanism (Savir and Tlusty, 2007). This robustness despite of noise which is also remarkable about DNA and embryological differentiation is the very signature of the $\mathrm{KB}$, since in its in-formational Hadamard matrix representation, one of its main properties is the fact that it is used as a basis for digital communications due to its robustness (Ahmed and Rao, 1975). This robustness to the influence of noise is apparent also in the KB topographic map of the sensorium in the human body (Rapoport, 2011b, Werner, 1968) and the visual recognition of arbitrary patterns that show an intrinsic universal gestalt, which is the $\mathrm{KB}$ (Ghrist, 2010; Carlson, 2009). 
We notice the striking similarity with the modification of the living matrix with regards to the environment, which we interpreted in terms of the KBL. In that work (Rapoport, 2011c) we contested the dualistic approach implicit to the rigid cathegorization of cell biology and phenotypical transformations derived from an Outside/Inside dual logic, which haemoglobin phagocytosis disproofs, yet is pervasive to biology, even in alledgedly radical revisions based on classical physics. Indeed, the $\mathrm{KB}$ has no separation between inside and environment, due to its self-containment; see Fig. 1 below. As a result, the substrate does not simply bind to a rigid active site; the amino acid side chains which make up the active site are molded into the precise positions that enable the enzyme to perform its catalytic function, evidencing a design-oriented change intrinsic to the present logophysical paradigm (Rapoport, 2011b,c) . IF may enhance the fidelity of molecular recognition in the presence of competition and noise via the conformational proofreading mechanism (Daily and Gray, 2009); yet, what we interpret as 'noise' might be interpreted as structured signaling, in a nested heterarchy of KBs, the HyperKlein Bottle, HKB (Rapoport 2010b, 2011b,c); this applies as well to evolution.

The topological embodiment of this coupling of an object (say, molecule, cell, etc) to the environment is difficult to identify since, as already explained, the integrity of these entities under study may change their structure beyond recognition; the same problem is encountered with molecules (Sokolov,1973). Yet,catenoid deoxyribonucleic acids in the mitochondria of several cells (catenanes are topologically-mechanically-interlocked molecular architectures, consisting of two or more interlocked macrocycles), are pervasive to organic chemistry and DNA (Schill and Zurcher, 1971).

A mechanism for the biogenesis of catenoid DNA on the basis of the MB and for the construction of MB DNA was proposed (Burdick, 1971). If a MB twisted $\eta$ times is cut along the midline, catenanes are formed whenever $\eta$ is an even number; when $\eta$ is unity, a single ring is obtained with a double circumference; other odd values of $\eta$ yield various knots (Bonchev and Rouvray, 2000;
Flapan,2000). The replication of MB DNA, which is equivalent to this slitting, naturally yields catenoid and knotted macromolecules. Topological operations on DNA are the core of cutting-edge synthetic nanobiology technologies (Seeman, 1998) and in metamaterials (Han et al., 2010). Through topological and self-assembling operations upon DNA material, catenanes, rotaxanes and knots are produced (Zheng et al, 2009), in which tensegrity structures having multivalued logic enantiomer configurations (as in Figure 2), have a central role. Hereon, we shall deal with the topologies and informatics of DNA in the setting of topological stereochemistry and the KB.

\section{The Klein Bottle Logic, Topological Stereochemistry of the Genetic Code and Informatics}

We shall retake the $\mathrm{KBL}$, as presented in (Rapoport, 2011c), to construct the Genetic Code. We depart from a logical-numerical representation of the KBL in Figure no. 1 in which we have identified four states by assigning 0 with Outside, 1 with Inside, so that the states are: Outside-Outside, which we write as 00, the Inside-Inside, 11, and two transitional states arising from selfpenetration, Outside-Inside, O1, and InsideOutside. This does not conform a dual logic.

Outside-Outside: 00

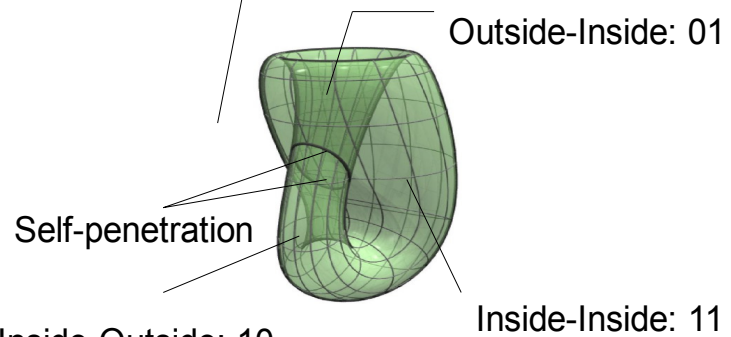

Inside-Outside: 10

Inside-Inside: 11

Figure 1. The Klein Bottle Logic (KBL), and its four states.

Indeed, we think of the above elements, $\mathrm{ab}$, as ordered pairs $[\mathrm{a}, \mathrm{b}]$, say the elements $\mathrm{OO}=[0,0]:=\mathbf{o},[1, \mathrm{O}]=\mathbf{i},[0,1]=\mathbf{j}, 11=[1,1]=$ 1, with the definitions $[\mathrm{a}, \mathrm{b}]+[\mathrm{c}, \mathrm{d}]=$ $[\mathrm{a}+\mathrm{c}, \mathrm{b}+\mathrm{d}],[\mathrm{a}, \mathrm{b}] \mathrm{x}[\mathrm{c}, \mathrm{d}]=[\mathrm{axc}, \mathrm{bxd}],[\mathrm{a}, \mathrm{b}]^{\prime}=$ [b', a'], with (a')' =a, axa $=a, a+a=a$ for all $a=0$ or 1 , the latter sum as usual differs from modulo 2, and a' is the operation of a changing side of the boundary of selfpenetration, hence: $0^{\prime}=1,1^{\prime}=0$, as if selfpenetration would not be the origin of the 
boundary, i.e., Aristotelian-Boolean logic. Then $\mathbf{i}^{\prime}=\mathbf{i}, \mathbf{j}^{\prime}=\mathbf{j}$, and $\mathbf{i x j}=\mathbf{o}$, so that $\mathbf{i} \& \mathbf{j}$ are non-trivial nilpotents. Thus, we have mapped the topological states of the KB into a 4-state de Morgan algebra which is not trivial since Outside, $o$, is different to Inside, 1 (Kauffman, 1978, 1989). The latter is, of course, the fundamental metacognitive assumption of science.

This is a new representation for the KBL to the one derived in (Rapoport 2011a), from which Matrix Logics -that has quantum fuzzy and Boolean logics as subcases- is derived (Rapoport 2009, 2011a, b, Stern, 2001). We notice that $\mathbf{i}$ and $\mathbf{j}$ are the imaginary time-waves (Kauffman 1978, 1987; Rapoport 2010a) that appeared as imaginary logical values in the Calculus of Distinctions (Spencer-Brown, 2010); we here see explicitly their association with the $\mathrm{KB}$ self-penetration. They are crucial to embryology (Rapoport, 2011c).

In topological phenomenological philosophy (Rosen, 2008), three states were considered: the container (Outside-Outside) that no longer corresponds to the Cartesian exterior space where objects are passively contained as Inside-Inside (the contained), which is the usual take on space, and the uncontained realizing the depth dimension of self-penetration, associated to time (Rapoport 2010b, 2011a,b). An identical 3state logic was provided (Varela, 1979), in which there is a single reentrance of the form on itself, archetypical Ouroboros. Yet, the distinction between the two states of selfpenetration transiting between Outside and Inside, according to which is the departing state, renders the direction of selfpenetration a necessary distinction by itself accounted by $\mathbf{i}$ and $\mathbf{j}$.

We relate this 4-state logic to the four letters, A, T (or U), G and C, of the GC. We follow a combinatoric-algebraic approach (Petoukhov and He, 2010), by considering the 2x2 matrix/table

\begin{tabular}{|c|c|c|}
\hline & 0 & 1 \\
\hline 0 & $\mathrm{C} 00 \quad(0)$ & $\begin{array}{lll}A & 01 & \text { (1) }\end{array}$ \\
\hline 1 & $\mathrm{U} 10 \quad$ (1) & G 11 (2) \\
\hline
\end{tabular}

Table 1. KBL Genetic codification

which we denote as [C, A; U, G], or still, P(1). We have written in parentheses the decimal interpretations of the elements of the logic; while the pairs $00,01,10,11$ will be interpreted in the following -for computational reasons- as binary numbers rather than elements of the de Morgan algebra. We shall introduce another distinctions that will be crucial to the topological theory of the GC. We know from (Rapoport 2011a, b, c) that the invocation of a distinction, is tantamount to invoke through the self-entrance of a form produced by this distinction (as a boundary/cleavage): i.e., a KB. In fact as we shall be considering three distinctions, bringing to manifestation a hyperKlein Bottle (HKB). They are produced by three subalphabets of the GC (Ycas, 1969), introduced in an algebraiccombinatorial framework for the GC (Petoukhov and He, 2010), in terms of pairs of attributes-antiattributes, described succintly in Fig. 2 below and by the following subalphabets:

Subalphabet No.1: o will code for pyrimidines (one ring in a molecule), 1 will code for non-pyrimidines, i.e. purines (two rings in a molecule), transcribed by $\mathrm{C}=\mathrm{U} / \mathrm{T}$ $=\mathrm{O}, \mathrm{A}=\mathrm{G}=1$.

Subalphabet No.2: amino-mutating or non-amino-mutating under action of nitrous acid HNO2 (Ycas,1969) the same division is given by the attributes "keto"or "amino", so that o stands for a letter with amino-mutating property (amino), 1 a letter without it (keto), $\mathrm{C}=\mathrm{A}=\mathrm{O}, \mathrm{G}=\mathrm{U} / \mathrm{T}=1$.

Subalphabet No.3: o a letter with three hydrogen bonds, 1 a letter with two hydrogen bonds; $\mathrm{C}=\mathrm{G}=0, \mathrm{~A}=\mathrm{U} / \mathrm{T}=1$; this is the usual subalphabet.

These distinctions introduces further multivaluedness in the topological codification of the KBL, -yet we shall not tag them with a symbol to distinguish which is the subalphabet they stand for- treating them as binary numbers so that we take 0 (Outside), 1 (Inside); their multivaluedness with respect to the subalphabets will manifest in the KB and HKB that will appear in the GC. In the sequel, the original interpretation of the matrix elements of $\mathrm{P}(1)$ by ordered pairs, say $\mathrm{C}=$ oo (OutsideOutside), will correspond to the concatenation of the first digit corresponding to No.1, the second digit to No.2. Thus already we have introduced inside the $\mathrm{KB}$ additional $\mathrm{KB}$ distinctions, an $\mathrm{HKB}$ as nested KBs, evidencing a polysemic and 
polysemantic character of the $\mathrm{GC}$ as a heterarchy composed by the $\mathrm{KB}$ associated to different subalphabets indicating the codification of distinct characters. This is illustrated in Figure 2 below. Recalling our previous discussions on the coexistence of orientable and non-orientable topologies for molecules (Sokolov, 1973; Herges, 2006), we shall see next that these subalphabets, similarly, produce the same effect for the GC. We consider the $4 \times 4$ matrix, $\mathrm{P}(2)=$ $[\mathrm{C}, \mathrm{A} ; \mathrm{U}, \mathrm{G}](2)$, the two-fold tensor (Kronecker) self-product of [C,A,U,G], i.e. $\mathrm{P}(2)=[\mathrm{CP}(1), \mathrm{AP}(1) ; \mathrm{UP}(1), \mathrm{G} \mathrm{P}(1)]$ ([8])which we write as a table (No.2):

\begin{tabular}{|c|c|c|c|c|}
\hline & $\begin{array}{c}\mathrm{C} \\
00(0)\end{array}$ & $\begin{array}{l}A \\
01(1)\end{array}$ & $\begin{array}{l}U \\
10(2)\end{array}$ & \begin{tabular}{|l}
$G$ \\
$11(3)$
\end{tabular} \\
\hline $\begin{array}{ll}\mathrm{C} \\
\mathrm{Oo}(0) \\
\end{array}$ & $\begin{array}{l}\text { CC } \\
0000(0)\end{array}$ & $\begin{array}{l}\text { CA } \\
0001(1)\end{array}$ & $\begin{array}{l}\text { AC } \\
0010(2)\end{array}$ & \begin{tabular}{|l|} 
AA \\
$0011(3)$
\end{tabular} \\
\hline A & $\begin{array}{l}\text { CU } \\
0100(4)\end{array}$ & $\begin{array}{l}\text { CG } \\
0101(5)\end{array}$ & $\begin{array}{l}A U \\
0110(6)\end{array}$ & \begin{tabular}{|l} 
AG \\
$0111(7)$
\end{tabular} \\
\hline $\begin{array}{l}\mathrm{U} \\
10(2) \\
\end{array}$ & $\begin{array}{l}\text { UC } \\
1000(8)\end{array}$ & $\begin{array}{l}\text { UA } \\
1001(9)\end{array}$ & $\begin{array}{l}\text { GC } \\
1010(10)\end{array}$ & \begin{tabular}{|l} 
GA \\
$1011(11)$ \\
\end{tabular} \\
\hline \begin{tabular}{|l}
$G$ \\
$11(3)$
\end{tabular} & $\begin{array}{l}\text { UU } \\
1100 \text { (12) }\end{array}$ & $\begin{array}{l}\text { UG } \\
1101 \text { (13) }\end{array}$ & $\begin{array}{l}\mathrm{GU} \\
1110 \text { (14) }\end{array}$ & \begin{tabular}{|l|} 
GG \\
1111 (15)
\end{tabular} \\
\hline
\end{tabular}

Table 2. We present $P(2)=[C P(1), A P(1) ; U P(1), G P(1)]$, the 2 -fold tensor product of the KBL genetic codification given by Table No. 1

We note again the numbering both by decimals in parentheses, while the duplets have their first two digits given by the rows codified by No.1 and the final two digits coming from the columns codified by No2, as in the original KBL genetic codification in Table No.1. We compute the 3 -fold tensor self-product, $\mathrm{P}(3)=[\mathrm{C}, \mathrm{A}, \mathrm{U}, \mathrm{G}](3)=[\mathrm{CP}(2)$, $\mathrm{AP}(2) ; \mathrm{UP}(2), \mathrm{GP}(2)$ ], i.e. the $8 \times 8$ genomatrix (Table No.3):

\begin{tabular}{|c|c|c|c|c|c|c|c|c|}
\hline & $\begin{array}{l}000 \\
(0)\end{array}$ & $\begin{array}{c}001 \\
(1)\end{array}$ & $\begin{array}{l}010 \\
(2)\end{array}$ & $\begin{array}{l}011 \\
(2)\end{array}$ & $\begin{array}{l}100 \\
(3)\end{array}$ & $\begin{array}{l}101 \\
(4)\end{array}$ & $\begin{array}{l}110 \\
(5)\end{array}$ & $\begin{array}{l}111 \\
\text { (7) }\end{array}$ \\
\hline $\begin{array}{l}000 \\
(0)\end{array}$ & $\begin{array}{l}\mathrm{CCC}(0) \\
00000 \text { Pro }\end{array}$ & $\begin{array}{l}\text { CCA(1) } \\
\text { 000001Pro }\end{array}$ & $\begin{array}{l}\mathrm{CAC}(2) \\
000010 \mathrm{His}\end{array}$ & $\begin{array}{l}\mathrm{CAA}(3) \\
000011 \mathrm{Gln}\end{array}$ & $\begin{array}{c}\text { ACC (4) } \\
000100 \mathrm{Thr}\end{array}$ & $\begin{array}{c}\text { ACA(5) } \\
000101 \mathrm{Thr}\end{array}$ & $\begin{array}{l}\mathrm{AAC}(6) \\
000110 \text { Asn }\end{array}$ & $\begin{array}{l}\text { AAA(7) } \\
000111 \text { Lys }\end{array}$ \\
\hline $\begin{array}{l}001 \\
(1)\end{array}$ & $\begin{array}{l}\mathrm{CCU}(8) \\
001000 \text { Pro }\end{array}$ & $\begin{array}{l}\text { CCG(9) } \\
001001 \text { Pro }\end{array}$ & $\begin{array}{l}\mathrm{CAU}(10) \\
001010 \text { His }\end{array}$ & $\begin{array}{l}\text { CAG(11) } \\
001011 \text { Gln }\end{array}$ & $\begin{array}{c}\mathrm{ACU}(12) \\
001100 \mathrm{Thr}\end{array}$ & $\begin{array}{c}\text { ACG(13) } \\
001101 \mathrm{Thr}\end{array}$ & $\begin{array}{l}\text { AAU(14) } \\
001110 \text { Asn }\end{array}$ & $\begin{array}{l}\text { AAG(15) } \\
001111 \text { Lys }\end{array}$ \\
\hline $\begin{array}{l}010 \\
(2)\end{array}$ & $\begin{array}{l}\text { CUC(16) } \\
010000 \text { Leu }\end{array}$ & $\begin{array}{l}\text { CUA(17) } \\
010001 \text { Leu }\end{array}$ & $\begin{array}{l}\text { CGC(18) } \\
010010 \text { Arg }\end{array}$ & $\begin{array}{l}\text { CGA(19) } \\
010011 \text { Arg }\end{array}$ & $\begin{array}{c}A U C(20) \\
010100 \text { lle }\end{array}$ & $\begin{array}{c}\text { AUA(21) } \\
010101 \text { Met }\end{array}$ & $\begin{array}{l}\text { AGC(22) } \\
010110 \text { Ser }\end{array}$ & $\begin{array}{l}\text { AGA(23) } \\
010111 \text { Stop }\end{array}$ \\
\hline $\begin{array}{l}011 \\
(3)\end{array}$ & $\begin{array}{l}\text { CUU(24) } \\
011000 \text { Leu }\end{array}$ & $\begin{array}{l}\text { CUG(25) } \\
011001 \text { Leu }\end{array}$ & $\begin{array}{ll}\text { CGU(26) } & \\
0110110 \quad \text { Arg } \\
\end{array}$ & $\begin{array}{l}\text { CGG(27) } \\
011011 \text { Arg }\end{array}$ & $\begin{array}{c}\mathrm{AUU}(28) \\
0111000 \mathrm{lle}\end{array}$ & $\begin{array}{c}\text { AUG(29) } \\
0111001 \text { Met }\end{array}$ & $\begin{array}{l}\text { AGU(30) } \\
011010 \text { Ser }\end{array}$ & $\begin{array}{l}\text { AGG(31) } \\
011011 \text { Stop }\end{array}$ \\
\hline $\begin{array}{l}100 \\
(4)\end{array}$ & $\begin{array}{l}\text { UCC(32) } \\
011100 \text { Ser }\end{array}$ & $\begin{array}{l}\text { UCA(33) } \\
011101 \text { Ser }\end{array}$ & $\begin{array}{l}\text { UAC(34) } \\
011110 \mathrm{Tyr}\end{array}$ & $\begin{array}{l}\text { UAA(35) } \\
011111 \text { Stop }\end{array}$ & $\begin{array}{c}\mathrm{GCC}(36) \\
100000 \mathrm{Ala}\end{array}$ & $\begin{array}{c}\mathrm{GCA}(37) \\
100001 \mathrm{Ala}\end{array}$ & $\begin{array}{l}\text { GAC(38) } \\
100010 \text { Asp }\end{array}$ & $\begin{array}{l}\text { GAA(39) } \\
100011 \text { Glu }\end{array}$ \\
\hline $\begin{array}{l}101 \\
(5)\end{array}$ & $\begin{array}{l}\text { UCU(40) } \\
101100 \text { Ser }\end{array}$ & $\begin{array}{l}\text { UCG(41) } \\
101101 \text { Ser } \\
\end{array}$ & $\begin{array}{l}\text { UAU(42) } \\
101110 \mathrm{Tyr}\end{array}$ & $\begin{array}{l}\text { UAG(43) } \\
101111 \text { Stop }\end{array}$ & $\begin{array}{c}\mathrm{GCU}(44) \\
101100 \text { Ala }\end{array}$ & $\begin{array}{c}\text { GCG(45) } \\
101101 \text { Ala }\end{array}$ & $\begin{array}{l}\text { GAU(46) } \\
101110 \text { Asp }\end{array}$ & $\begin{array}{l}\text { GAG(47) } \\
101111 \mathrm{Glu}\end{array}$ \\
\hline $\begin{array}{l}110 \\
(6)\end{array}$ & $\begin{array}{l}\text { UUC(48) } \\
110000 \text { Phe }\end{array}$ & $\begin{array}{l}\text { UUA(49) } \\
110001 \text { Leu }\end{array}$ & $\begin{array}{l}\text { UGC(50) } \\
110010 \text { Cys }\end{array}$ & $\begin{array}{l}\text { UGA(51) } \\
110011 \text { Trp }\end{array}$ & $\begin{array}{c}\text { GUC(52) } \\
111000 \text { Val }\end{array}$ & $\begin{array}{c}\text { GUA(53) } \\
111101 \mathrm{Val}\end{array}$ & $\begin{array}{l}\text { GGC(54) } \\
111110 \text { Gly }\end{array}$ & $\begin{array}{l}\text { GGA(55) } \\
111111 \text { Gly }\end{array}$ \\
\hline $\begin{array}{l}111 \\
(7)\end{array}$ & $\begin{array}{l}\text { UUU(56) } \\
111000 \text { Phe }\end{array}$ & $\begin{array}{l}\text { UUG(57) } \\
111001 \text { Leu }\end{array}$ & $\begin{array}{l}\text { UGU(58) } \\
111010 \text { Cys }\end{array}$ & $\begin{array}{l}\text { UGG(59) } \\
111011 \text { Trp }\end{array}$ & $\begin{array}{c}\text { GUU(60) } \\
11110 \text { Val }\end{array}$ & $\begin{array}{c}\text { GUG(61) } \\
111101 \mathrm{Val}\end{array}$ & $\begin{array}{l}\text { GGU(62) } \\
111110 \text { Gly }\end{array}$ & $\begin{array}{l}\text { GGG(63) } \\
111111 \text { Gly }\end{array}$ \\
\hline
\end{tabular}

Table 3. The KBL codification of the 64 codons and their aminoacids (written in abbreviated form) and stop codons, and the bioinformatic codification -as in Petoukhov and He (2010), here introduced in terms of the matrix given by the 3-fold tensor product of the KBL codification of the genetic code given in Table 1.

In Table no. 3 we have represented the 64 codon-anticodon triplets in which we have also written their decimal (in parenthesis) and binary representations, and written the abbreviations for the aminoacids synthetized by them. Each of the 64 triplets has been individualized uniquely by a number consisting of the concatenation of six binary digits, the first three coming from the rows correspond to the No.1 codification, while the last three binary digits provided by the corresponding column codifies according to No.2; for example, triplet CAU is codified by the binary number 001010, where the first three digits 001 corresponds to the No.1 assignment for CAU whilst the last three digits 010, corresponds to the No.2 assignment; the decimal notation for the concatenation 001010 is 10. Remarkably, each pair codon-anticodon (and only such pair) has the sum of their decimal numbers equal to 63 (111111, in binary notation), say CAU which is 10 its anticodon GUA has the decimal number 63. We note that No.3 transcriptions of $\mathrm{C}$ with $\mathrm{G}$, and A with $\mathrm{U}(\mathrm{T})$, are completely determined by the other two subalphabets, as shown in Fig. 2 below, and correspond to the mutual transcriptions of 
Outside-Outside/ Inside-Inside, and of the time waves Outside-Inside/Inside-Outside, and they correspond to the binaryopposition attribute by which the former (latter) correspond to three (two) hydrogen bonds. This genomatrix has surprisingly rich symmetry properties which invite to topological interpretations, previously unaccounted for, which we shall realize next.

They further indicate relations with hypernumbers and 8-fold quark-like structures which also appear in Matrix Logic (Rapoport, 2011a) and NUCRS, 5-fold Fibonacci structures (also essential to both NUCRS and torsion fields), and in particular, a music translation of the GC, in an epochal unified approach (Petoukhov and He, 2010).

Firstly, we have both symmetries along the rows \& columns due to No.1 \& No.2, respectively, and thus we have, with respect to them, an associated 2-torus; see Fig. 2 below. We note that the columns correspond to the classical octets reflecting biochemical properties of elements of the GC (Ycas, 1969). Secondly, it is bisymmetric (with respect to No.3), i.e. symmetric with respect to both diagonals, say UUC which is the matrix element corresponding to 7 th line and first column has the anticodon AAG in 7 th line and 1st column. Hence, we have a $\mathrm{MB}$ produced by $180^{\circ}$ rotation about the central line that divides the genetic code into codon-anticodon sectors, so that superposed on the non-orientable topology, we have all the codon-anticodon pairs, with each codon having its superposed pair that can be thought as positioned on the 'other' side of the band; say we have UUC, UUA, UGC \& UGA superposed to AAG, AAU, ACG \& ACU, respectively. This is the MB topology of the genomatrix $\mathrm{P}(3)$.

If we further consider now the (No.2wise) column symmetry, we finally obtain a $\mathrm{KB}$. Yet, it is more than a single $\mathrm{KB}$, but four of them, produced by the superposed 1st/8th, 2nd/7th, 3rd/6th, 4th/5th columns, with the first element of each superposition inverted with respect to the second,yet embedded in a single $\mathrm{KB}$ given by the 64 triplets: an HKB. Finally we can use the row No.1 subalphabet to produce a folding of the genomatrix along its horizontal middle line, which further using the diagonal bisymmetry we produce a second HKB with four others embedded given now by the superposed rows 1st/8th, 2nd/7th, 3rd/6th, 4th/5th, with the same inversion as before.
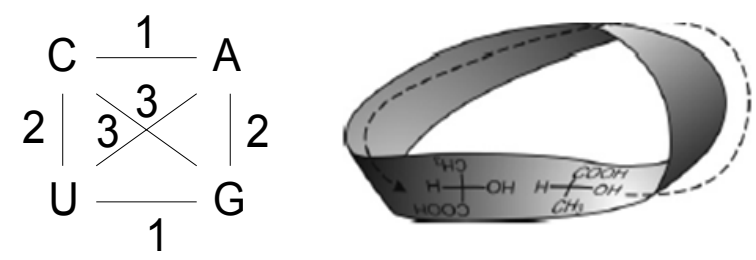

Figures 2 and 3. In Fig.2 (Petoukhov and He, 2010) the lines stand for transcription and the subalphabet by which each operates is the number attached to it. Remarkably, it also presents the symmetries of genomatrixes for coding sequences of arbitray length, and their topologies. Folding for topological identification according to these symmetries, say No3. yields MB of both chiralities, which followed by either No.2 or No.1 yields the KB; the combination of No.1 with No.2 yields a 2-torus. In the right hand side of the $\mathrm{MB}$ Fig. 3, we have drawn the Fischer formula for D-lactic acid which if we continue on the surface to the 'other' side we obtain the L-form drawn displaced to the left for allowing its vision; for the genomatrix we have instead the four superpositions of either the pairs of opposed rows (columns) with say each first element opposed to the second drawn on the surface which is a quantum interface. For the Mendeleev table we have instead a superposition of 'matter/antimatter'-atom, in each side (Boeyens, 2005); a continuous transformation changes matter into antimatter, and viceversa. The inversion of each element of a pair of rows or columns mandated by taking No3., plays the role of the antiparallelism in the $\mathrm{DH}$.

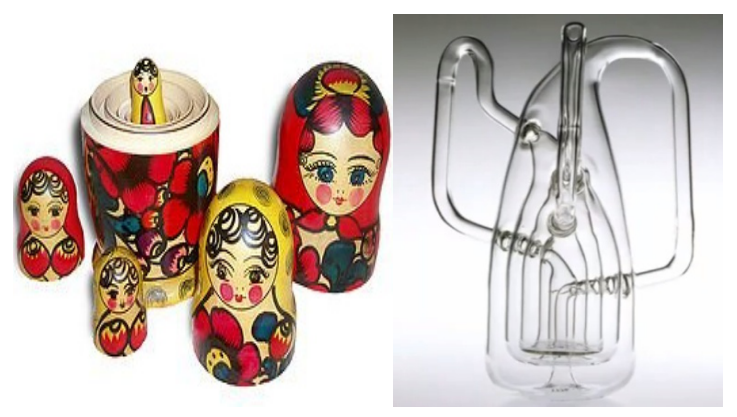

Figure 4. Two possible paradigms of inter-relation heterarchy systemics. In the Ihs we find the Matrushkas: Recursive containment without self-contention nor reentrance of the Whole on the Parts; this is the current paradigm. In the rhs we have a completely different conception. This is a view of an exemplar of the hyperKlein Bottle- HKB (courtesy of the Science Museum, London; Creative Commons). The systems reenter on themselves and may also reenter in others. The former paradigm is a reduction of the latter by erasing all reentrances. This is a suppression of complexity, in the etymological sense of folding, so that a first attempt to describe the emergentist paradigm is that it erases that which will later differs to a metalogophysics that runs by the name of Paradigm of Complexity! 
A simple version of an $\mathrm{HKB}$ with only one $\mathrm{KB}$ recursively iterated, (instead of four) is given in Fig. no. 4

We have thus found two fractal $\mathrm{HKB}$ structures in the genomatrix $\mathrm{P}(3)$, and recursively in $\mathrm{P}(\mathrm{n})=[\mathrm{CP}(\mathrm{n}-1), \mathrm{AP}(\mathrm{n}-1)$; $\mathrm{UP}(\mathrm{n}-1), \mathrm{GP}(\mathrm{n}-1)]$, for arbitrary $\mathrm{n}$ naturalaccording to the choices of No.1/No.3, i.e. the choice of attributes pyramidinepyrimine/hydrogen atoms, and No.2/No.3, i.e. amino-keto/hydrogen atoms, in the GC arising from the KBL, and we also have a 2torus by using No1. \& No.2; as we can easily visualize from the definition of the tensor product, it produces the fractality which reproduces the original (i.e., $\mathrm{n}=1$ ) topological identification introduced in Fig.1.We remark again, this has surfaced from a simultaneous double interpretation which is both perceptual, conceptual and operational- i.e., establishing and reading three subalphabets for transcription, which combined in pairs produces the remaining one; this trascends the usual approach to the genetic code as well as the combinatorial one (Petoukhov and He, 2010); these topologies apply as well to the codification of the sequences of $\mathrm{n}$ letters by $2 \mathrm{n}$ digits.

The information content in each interior $\mathrm{KB}$ to the $\mathrm{HKB}$ is not the same as the one contained by its neighbours. Also, in the transition from $\mathrm{P}(2)$ to $\mathrm{P}(3)$ or, more generally, from $P(n-1)$ to $P(n)$, in which the latter represents $n$-plets with $2 n$ binary digits, with the first $\mathrm{n}$ digits codifying subalphabet No.1, the last $\mathrm{n}$ digits codifying subalphabet No.2, there is an embedding so that the information of the (n-1)-plets is carried into the n-plets, as a kind of memory of self-referential action (selfmultiplication). Again, $\mathrm{P}(\mathrm{n})$, for arbitrary $\mathrm{n}$, also presents the same symmetries of $\mathrm{P}(\mathrm{n}-1)$, and ultimately those of $\mathrm{P}(2)$, and thus we found the same coexistence of topologies of the genomatrix, according to which of the three pairs of attributes are considered, for n-plets of arbitrary length. We have thus unveiled in the GC the same situation of polytopologies (we recall that also DNA is polygeometrical) that appears already in topological stereochemistry (Sokolov, 1973) which we claimed to be essential to cell biology and to embryological development, and a fortiori, to evolution (Rapoport, to appear, and 2011c). If one should construct the catalog of genetic sequences of various lengths and composition, it can be done on the basis of the described natural system of numbering the sequences as multiplets. All n-plets, which begin with one of the four letters C, A, U, G, are disposed in one of the four quadrants of an appropriate genomatrix $\mathrm{P}(\mathrm{n})$ because of the specifics of tensor multiplication.

Thus, the codon-anticodon sequence of arbitrary length $\mathrm{n}$, when considering pairs of subalphabets, corresponds to a path on either two fractal $\mathrm{HKB}$, or a 2-torus, given by $\mathrm{P}(\mathrm{n})$. This construction does not require the assumption of the double helix (DH); the latter is bound to one single subalphabet which is already evident in the No.3 reading of $\mathrm{P}(\mathrm{n})$ which instead yields a MB.A MB model for circular genetic code was proposed (Burdick,1971), yet to our knowledge, this has not been the case for a KB structure (Bonchev and Rouvray, 2000; Flapan, 2000). These findings, which in our present discussion has neglected the topology of the p-orbitals of aromatics crucial to DNA, support the unifying paradigm for chemistry (Bonchev and Rouvray, 2000; Herges, 2006; Sokolov 1979) that claims that the topology of molecules, rather than their hybridization geometry relations, characterizes their stereochemical configuration, which we have suggested to be crucial to allosterics, cell biology and embryological differentiation (Rapoport, to appear \& 2011c). Yet, the torsion geometry of the wave functions plays a central role also in defining the shape (Boeyens, 2005).

A KB model of DNA, may explain why only a single 5'-3' polymerase has been found so far, so that the antiparallel 3'-5' invoked by the $\mathrm{DH}$, was early in the history of the GC claimed to be unnecessary for transcription for closed DNA (Burdick, 1971), a particular case to the one here unveiled. We recall that the two strands that make up the $\mathrm{DH}$, each have a stereochemical orientation -the socalled 5'-3'- orientation, by which each phosphate group in a strand joins the 5 ' carbon of one sugar to the 3 ' carbon of the next. This orientation must be the same for every phosphate group within a strand, which imparts a directionality to the strand as a whole. The two strands of the B-form duplex are oriented so their 5'-3' directions are antiparallel in the DH. Consequently, 
DH DNA molecules can be closed into a circle only by joining together the ends of each of the two individual strand. Circularization by joining the ends of two strands to form the MB is forbidden because the bonds required would violate the conservation of 5'-3' directionality (Benham, 1995). All that said, this claimed to-be Nature's prohibition appears not to have been realized remarking the topological being of stereochemistry, rather than the geometrical double helix model (Kurosawa and Okazaki, 1975; Yudelvich et al., 1968; Yoshikazu et al, 1975). Starting with DNA material and through folding and "sticky ends" (i.e., single strands, consistently with the present findings and (Burdick, 1971), opposite chirality MBs have been produced (Han et al, 2010l) and through joining their sides the $\mathrm{KB}$ can be realized; paradoxically, the DNA model advocated by this authors is the DH. So in principle, a biochip that may embody the KBL as the logic for quantum computation with self-correcting codes (Stern, 2001), is reachable. We note that the crossover effect present at the core of the KB and consequently in the GC, is at the basis of morpho-functional structures in the human organism, such as the crosswise connection of brain hemispheres with the left and the right halves of a human body, of chromosomes, the crosswise gestalt of optic nerves from eyes in the brain (Annet, 1985), visual hemilateral synchronization (Rapoport 2010b, 2011b).

Remarkably, in terms of torsion shear elastodynamics, it is easy to see that the double helix can be easily obtained from a single elastic rod, to which we apply torsion, so that in approaching the two ends of the band, two helical structures appear without breaking the band, and further torsioning still produces four helixes, and so on. These are stable configurations (Goriely and Tabor, 1997; Goriely, 2001), and appear as the basis for the torsion geometry of biological structures and functions; for further references (Rapoport, 2001c).

There is another HKB fractal structure for the genetic code that is produced departing from another matrix representation for the $\mathrm{KB}$, namely we consider the Hadamard matrix $\mathrm{H}(2)=$ $[\mathrm{C}, \mathrm{A}, \mathrm{U}, \mathrm{G}]=[1,1 ;-1,1], \mathrm{H}(4)$ and $\mathrm{H}(8)$, the 2 and 3-fold tensor self-product of $\mathrm{H}$, respectively,

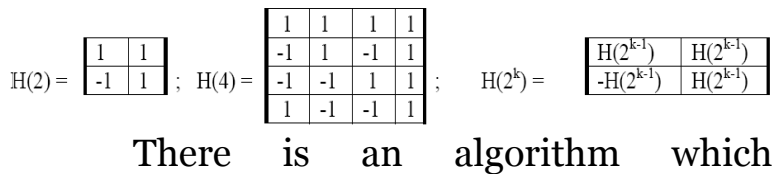
transforms $\mathrm{P}(3)$ into $\mathrm{H}(8)$ and from $\mathrm{P}(\mathrm{n})$ to $\mathrm{H}\left(2^{\mathrm{n}}\right)$ (Petoukhov and $\mathrm{He}, 2 \mathrm{O} 10$ ), and thus from two different topological codifications arising from the $\mathrm{KB}$, we obtain the same genomatrix representations of the GC. While in the former approach the HKB was apparent, this is no longer the case for $\mathrm{H}(8)$. This minimalistic topological basis for the genetic code appears to be related to its marvelous resistance to environmental hazards. Indeed, the Hadamard matrix approach yields mosaic fractal structures with 32 positive and 32 negative ones, which are associated to Rademacher functions (which only take +1 and -1 values) from the digital theory of signal processing. Hadamard matrices, which play a crucial role in the matrix realization of the KBL and its relation with quantum logic (Rapoport 2011a, b; Stern, 2001) and in quantum computation, are used widely in the theory of coding, being crucial to the robustness of transfer of digital information with regards to environmental noise (Ahmed and Rao, 1975), and thus the KB not only provides the basic codification, but also the robustness of the GC and a fortiori, that of embryological development.

Indeed, we return now to the codification of the differentiation waves in embryological development introduced in (Rapoport, 2011c).

Recalling the characterization of the latter in terms of contraction -say C (or alternatively expansion, E) waves followed by the imaginary transitions of $\mathrm{C}$ to $\mathrm{E} \&$ the closing transition $\mathrm{E}$ to $\mathrm{C}$ (with the same alternative substitution everywhere), altogether forming one instance of differentiation, codified as the binary sequence 000110, where the first two digits oo codify the $\mathrm{C}$ wave, the second two digits o1 codify the imaginary logical state of transition $\mathrm{C}$ to $\mathrm{E}$, concluded by the final digits 10 of the self-penetration of the dividing boundary between distinct types of tissues, due to the imaginary transition from $\mathrm{E}$ to $\mathrm{C}$. We retrieve here the topological 
representation of the KBL, now for embryology. In the present rendering, this corresponds to a unique triplet, AAC (asparagine), and its anticodon, UUG (Leucine), codified as 111001, corresponds to the alternative wave initiated by an E-wave. Asparagine is known to supress the growth of leukemic cells (Li et al, 2006). Leucine appears in the germination of grains related to the increase of metabolic activity and to control some of the biochemical events at meiotic prophase and to appear in the germination of grains related to the increase of metabolic activity (Sopanen et al, 1980).

Also the Fibonacci sequence can be introduced in the present framework- We take a corresponding multiplet of the matrix [C A; U G](n) and change its letters $\mathrm{C}$ and $\mathrm{G}$ to $\varphi$, the golden mean; instead of letters $A$ and $\mathrm{U}$ in this multiple we place $1 / \varphi$ (Petoukhov and He, 2010). As a result, we obtain a chain with $\mathrm{n}$ links, where each link is $\varphi$ or $1 / \varphi$; we recall that they are the eigenvalues of the OR \& NAND operators of the Matrix Logic derived from the KBL, so their appearance in the GC from the KBL is not accidental. In fact, OR and NAND are represented by the 2x2 matrices [0;1,1,1] and $[1,1 ; 1,0]$, respectively, which coincide with $[\mathrm{F}(0), \mathrm{F}(1) ; \mathrm{F}(1), \mathrm{F}(1)]$ and $[\mathrm{F}(1), \mathrm{F}(1) ; \mathrm{F}(0)$, $\mathrm{F}(1)]$, and the $n$-th power of OR \& NAND are [F(n-1),F(n); F(n),F(n+1)], [F(n+1),F(n); $\mathrm{F}(\mathrm{n}), \quad \mathrm{F}(\mathrm{n}-1)]$ respectively, with $\mathrm{F}(\mathrm{n})$ representing the $n$-th element of the Fibonacci sequence. So we are considering $[\mathrm{C}, \mathrm{A} ; \mathrm{U}, \mathrm{G}]=[\varphi, 1 / \varphi ; \varphi, 1 / \varphi]$. For further elaborations with the relations with the $\mathrm{CG}$, see (Petoukhov and He, 2010).

\section{The Klein Bottle, Bistable Perception, the Double Helix and the Non-dual Basis of the Dual Conception of Physics and the Genome}

Having produced the 64 codons at the third stage of recursion by tensor selfmultiplication, we proceed to examine the relations with the Code of Nature (CN) proposed for the $\mathrm{GC}$, in the setting of the NUCRS (Hill and Rowlands, 2008; Rowlands, 2008). This theory based on recursivity, i.e., algorithmic self-reference, establishes a symbolic relation between the Dirac algebra of quantum mechanics (thus the 64 triplets are mapped into the 64 basis elements of this algebra) and the GC, which is further embodied in a fractal structure produced in terms of the Platonic solids (PS).Thus, by following the construction of the $\mathrm{CN}$ and its association with the Dirac algebra, we may establish, an isomorphic representation between the KBL construction and the $\mathrm{CN}$.

The latter's construction is produced recursively starting from the tetrahedron; see Fig.no. 5 below: At each of its vertices the four letters, A, C, G and T/U are positioned, centered in the latter for distinction, similarly to the sign distinction for it in the Hadamard representation above). This single tetrahedron is taken to represent single stranded DNA from which through a fractal recursive construction, the GC follows in terms of fractal concatenations of tetrahedrons. Consequently, all the 64 triplet codons which are uniquely represented by the elements of the Dirac algebra can be positioned in the four triangle faces of the tetrahedron, which thus in principle contains the basis for the operation of GC defined by DNA or mRNA, whatever its topology and geometry may be. We recall that the KBL generation of the GC, has placed in evidence that the transcription does not require the $\mathrm{DH}$ since the two HKBs found in $\mathrm{P}(3)$ fully represents the transcription for triplets, and further for n-plets by $\mathrm{P}(\mathrm{n})$.

A usually unacknowledged property of the PS drawn in 2D projections centered in one of the vertices, is that their perception gives rise to the so-called bistable perception, i.e. two possible percepts connected in time arise, depending on the interpretation -provided by the other sides acting as cues- of the center being in the hind or the fore. Thus, time and depth, as the primeval dimension, produces this double being coexisting in time (Rapoport, 2010b, 2011a, b). This projection was devised in organic chemistry to Emil Fischer, to represent enantiomers in $2 \mathrm{D}$, and in topological stereochemistry is associated to the MB (Herges, 2006) and the KB (Figure no. 3).

Perception is the issue at stake with regards to $2 \mathrm{D}$ representation of the stereochemistry (we recall, stereo means solid, alluding to $3 \mathrm{D}$ ) of organic chemistry which produce a multivaluedness that a set of rules for "disambiguation" is convened to remove them (Brecher,1999,2006): 
Herewith, the two possible percepts that arise from the single DNA strand tetrahedron are to be rendered distinct, which the NUCRS, to represent the assumed $\mathrm{DH}$ transcription operation of the GC, choses to represent as a star (double interlocked) tetrahedron, see Figure no. 6 below, thus starting the construction of the $\mathrm{CN} \&$ the transcription in the GC: This is the duplication of $3 \mathrm{D}$ to the NUCRS and the $\mathrm{CN}$ bauplan of the GC, and entails a duplication of the $\mathrm{KB}$, which becomes now a HKB with two nested superposed KBs (simpler than Fig.no. 4), each associated to one of the tetrahedrons; this duplication has a common singularity by which these KBs reenter, the origin, T/U for DNA/RNA. The paradoxical perceptual effect of this duplication is the frustration of the multivaluedness, as shown in Figure no. 6 below, and the duality of the NUCRS and a fortiori, the $\mathrm{DH}$ and $\mathrm{CN}$, are produced (Hill and Rowlands, 2008; Rowlands, 2008).

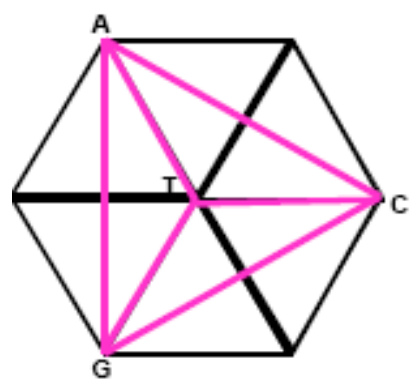

Figure 5. Single Tetrahedron (within a cube) representation of a single strand DNA (or mRNA by replacing $U$ for $T$ ) in the usual model. Reproduced from (Hill and Rowlands, 2008); courtesy of the authors.

The CN's algorithm starts by using a single tetrahedron in which the four bases are placed, each in a corner. Now the tetrahedron can now be considered to contain in an in-formation level, all the 64 possible triplet codons built before from the KBL, by codifying the four bases as in Table No.1, and further taking its 3 -fold tensor products.

The next step in the algorithm of $\mathrm{CN}$ is to superpose two tetrahedrons; the full line is for the 'positive' strand, the broken line for the 'negative strand', as in Figure no. 6. This produces the coupling of A-T (or U), G-C, as in the double helix model, or more fundamentally, from the KBL codification in Table no. 1, which biochemically is the representation in Subalphabet No. 3 in Fig. No. 2.

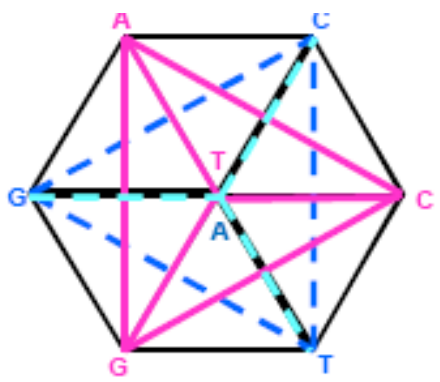

Figure 6. The Double Tetrahedron within a cube in the $\mathrm{CN}$, proposed to be the geometry of double stranded DNA, (or mRNA, by placing $U$ for $\mathrm{T}$ ) respectively; reproduced from (Hill and Rowlands, 2008); courtesy of the authors. In the $\mathrm{CN}, \mathrm{T}$ (or U) correspond to time, while A corresponds to space which are now interlocked; more of this below. Full and broken lines correspond to opposite directions of what is claimed to be two strands as in the double helix.

The perception of the single tetrahedron within a cube in Fig. no. 5 produces two figures in time according to the contextual interpretation of $\mathrm{T}$ (or $\mathrm{U}$, would we consider mRNA) being either the Outside or Inside vertex of the cube. This generation produced by zooming on the centre is the T-centered (e)invagination of the $\mathrm{KB}$ map of the sensorium and its generation is related to the existence of visual cues around $\mathrm{T}$ (Rapoport 2010b, 2011b). In the Double Tetrahedron, the zooming effect is frustrated and the perception in time is transformed into a spatial perception, the two interlocked KBs, conforming a HKB, or the Double Tetrahedron for the Double Helix (DH), in the CN's representation of the usual model.

By setting A \& $\mathrm{T}$ superposed in the origin, subalphabet No.3 is rendered as the identification of the Outside-Outside \& Inside-Inside of two superposed KBs in their self-penetration singularity, consistently with the dual perception of the (e)-inversion of them through the singularity, and thus the mutual transcription of $\mathrm{C}$ with $\mathrm{G}$ is also fixed as the identification of the Outside-Inside and Inside-Outside states. Thus two states are produced, one being a single imaginary state by identifying both imaginaries in the $\mathrm{KBL}$, the other being the identification of now Outside \& Inside; the latter identification is far from being the Cartesian Cut claim of reality. As for the $2 \mathrm{D}$ rendering of the PS and the ensuing fractal GC obtained in the $\mathrm{CN}$, in the NUCRS 2D has a 
generative (in fact genetic as the $\mathrm{CN}$ shows) function which is the case already of the $\mathrm{KB}$ surface: it is $2 \mathrm{D}$ as well as the $\mathrm{KBL}$ is represented in $2 \mathrm{D}$.

A fortiori, we find that the NUCRS is based in the HKB, and in particular the double $3 \mathrm{D}$ space of this theory functions as objectifying through the double-tetrahedron based generation of the $\mathrm{CN} \& \mathrm{GC}$, the multivalued character of the $\mathrm{KBL}$ and its hyperextension to HKB.

The bistable perception of the initial single tetrahedron representation of the four letters of the GC is mapped as two different distinguishable gestalts, the star (interlocking two) tetrahedron, yet producing a codification of the 64 (not 128) codons already in the genomatrix , which we stress does not require the assumption of the $\mathrm{DH}$ (this assumption is implicit in (Petoukhov and He, 2004, 2010), by which the interpretation of the bisymmetry of the genomatrix is done in terms of it). A fortiori, the triplets represented in the $\mathrm{CN}$ in the third order level star tetrahedron, which embeds the original pair of Figure no. 6 in a fractal structure which should be transformable to our previous construction, yield the same information (64 triplet codons and 20 amino acids) than the third tensor product of [C, A, U, G], i.e., $\mathrm{P}(3)$.

Yet, the latter's lack of assumption of the $\mathrm{DH}$, a fortiori shows the non necessity of the $\mathrm{DH}$ in $\mathrm{CN}$, would not be that a duplication of $3 \mathrm{D}$ is required by its fermionic based character, in which the fermion and the Universe couple through nilpotence selfreferentially in the Dirac algebra. In this coupling, the Universe is the context for the fermion; yet we remark that the fusion of context and content is an essential operation of the $\mathrm{KB}$, through the transitional states, i.e., time. Would we follow the suggestion in p.491, (Rowlands, 2008), of identifying the (torsion nilpotent boson, in our KB-based theory) singularity produced by the KB selfpenetration, with the fermion (which thus becomes the fusion of the Inside-Outside \& Outside-Inside states, i.e., A with $\mathrm{U}$ ), then the complement of the fermion - the Universe- for its algebraic self-annihilation returning to 0 , requires the fusion of the other two states, completing thus the transcription rules of No.3. Thus, time, as the process and depth generative dimension embodied in the reentrance of the KB by selfpenetration, the essential self-referential action, is objectified (materialized) as a double $3 \mathrm{D}$ space and the $\mathrm{CN}$ and the GC materialize as well through the double tetrahedron. This materialization seems to be the case in the 6D torsion geometry of the growth of molluscs (Illert, 1990,1992) which anticipate their unfolding; would the geometry be projected to $3 \mathrm{D}$, the molluscs would not be created: they would break. We have shown that the elasticity of the ovum allows for its eversion, and through the interlocking of pairs of $\mathrm{KBs}$, the turning inside-out of the ovum takes place and embryological development is produced (Rapoport, 2011c).

The construction of the NUCRS in terms of the Dirac algebra is quite remarkable in many aspects in view of the fact that the Dirac equation for one-half spinors is equivalent -not without restrictions to $2 \mathrm{D}$, that already appeared in the second quantization of the electromagnetic field- to the Maxwell equation of electromagnetism, i.e. an equation for a spin one field, a boson (Rapoport, 2005), and thus, conceptually, in principle, the NUCRS can be introduced starting with spin 1, rather than fermions.

Indeed, the $\mathrm{KB}$ is associated to spin 1

fields (Rapoport 2010a, 2011a, b, 2012; Rosen, 2008) specifically to the selfreferential photon torsion geometry associated to the eikonal (nilpotent, in the language of NUCRS, plenumpotent in the language of the present theory) equation for propagating light rays; no relation of the photon to the rest of the Universe, as is the case of NUCRS's fermion is required, because of its self-referential KB being.

In the NUCRS this duplication of $3 \mathrm{D}$ and the stress on fermions, unacknowledged its KBL origin and generically of selfreference not solely restricted to algorithmic recursion, entails the DH (with subalphabet No.3 transcription): Yet, the KBL construction of the GC produces topologically the transcription, without the $\mathrm{DH}$.

Thus, we reach the surprising perhaps- conclusion that the $\mathrm{DH}$ associated to fermions in the NUCRS \& $\mathrm{CN}$, is nothing else than a recourse to materialize time and self-penetration of the $\mathrm{KB}$-we recall, the 
material action of self-reference-, through a logical spatial 3D representation which embodies spatially the multivaluedness of the KBL. Remarkably, molluscs unfold through the self-iteration of single helixes (Illert, 1990, 1992).

As we already discussed, it is here that the NUCRS finds its dualistic base further manifested in the relation with the GC established by the CN.This is associated to the breaking of the 8-fold full symmetry to the 5 -fold symmetry in which mass and charge appear, as is case of the Dirac equation for a spin $1 / 2$ field, or its massless Maxwell equation equivalent in which the mass of the electron is related to rotational kinetic energy of the spin-plane of the spinor.

This claim of dualism as a basis for physics, at least as generated by NUCRS, is not the case, since it has become evident that it comes from duplicating a $\mathrm{KB}$ which is certainly not a dual logic nor principle.

With regards to the KBL and the ensuing polysemic codification of the GC, the $\mathrm{DH}$ is unnecessary; this appears again in the 2D hypercube representation of the genomatrix $\mathrm{P}(3)$ : two images, in time, appear of the 64 triplets, which would we wish to materialize as distinct, we would recourse to the construction of the $\mathrm{CN}$, yet nothing new would appear from this bauplan, which the hypercube does already represent without duplication of either $3 \mathrm{D}$ or $4 \mathrm{D}$ (Petoukhov and $\mathrm{He}, 2004)$. Indeed, the closed path in the hypercube joining the 64 triplets as vertices of a graph transversing each of them only once, but for the extremes, i.e. a Hamiltonian path, is unique. Remarkably, the reduction of the 64 Dirac algebra/codons to the 20 aminoacids, in the KBL construction of the $\mathrm{GC}$, brings to the fore symmetry properties of $\mathrm{P}(3)$, in which the Time operator of KBL (Rapoport, 2011a, b), and the establishment of chronomes (time cycles) is manifested; we shall elaborate this elsewhere.

\section{Conclusions and Extensions}

We have presented a proposal for the allosteric effect in terms of the same principle for embryological development, and still for the membrane and cell biology, and stereochemistry (Rapoport, to appear), in which shape determination is related to topological transformations that transform an orientable topology with a well defined interior and exterior two states, to nonorientable in which the logic is no longer dual, but the multistate KBL. Thus, the fusion of logic and physics, which we have called logophysics, which has quantum geometry as the physical correlate associated to light torsion quantum geometry, has been found to lie as a possible foundation common to biology, chemistry and also physics, the latter in account of the fundamental role of quanta and light.

A theory for the GC has been proposed departing from the $\mathrm{KBL}$ and its further multivalued representations of the stereobiochemical structures of the GC, to yield two HKB -and a 2-torusrepresentations which do not require the DH. This suggests the need of reconsideration of the transcription process. This polytopological character of biochemistry already appears in the conformational geometry of organic chemistry, specifically in aromatics and has claimed to be a new paradigm for stereochemistry.

In particular, we have suggested that allosterics may also be related to hypertopological accomodation (and quantum resonances) already apparent in hemoglobin phagocytosis; here the role of a quantum guidance which has a correlate as elastodynamics is the case.

The two representations of the GC in terms of HKBs and their biochemical alphabets can be thought as establishing a relation between the GC and the environment in which the latter is transformed into the GC and vice versa, since the KB produces this transformation in the first place, and we have shown in (Rapoport, 2011c) to be the case of the embryological development. In the other hand, the torus structure and its biochemical alphabet of the GC points out to a different way of trading between the $\mathrm{CG}$ and the environment, in which so to speak, the GC is isolated 'within' establishing a dual boundary with respect to the environment. In a metacognitive level, this points out to a higher order HKB in which both modes are possible. This metacognitive level speaks out about metaphorization, calling for what may 
appear, in first glance, as an unwarranted detour.

Let us discuss a characterization of metaphorization, in terms of an HKBLogic, a family of interprenetrating KBs. Indeed, to metaphorize requires that a subject establish a comparison between A (we called it, the metaphoron) and its alleged substitute B. In doing this, there is an identity transparency of the subject with the elements of the relation, and the relation itself is the case of this identity. So we are in the presence of three KBs, the subject, the elements of the relation of the metaphor, and the metaphor, and still a fourth one, the metaphorization, as a possibility that enacts the three previous elements.

If this metaphor is to be found valid, then other subjects will find meaning and sense in the metaphor, i.e., in the relation established by the subject, which has become partially identified with the metaphor and its elements. This commonness of logical value, is the logical state of a $\mathrm{KB}$ that contains all subjects and the elements of the metaphor, and the relation itself between the elements, so it is in fact a KB interpenetrated by other $\mathrm{KBs}$, an HKB as shown in Figure 4.

Some subjects will not necessarily assent to the meaning of the metaphor, may place it in doubt, suspending judgment, or reject it in the first place; we all know that the modes of sense of humor are culturally bounded, while a sense of humor is a personal, ethnic or social trait and all of them together. So here we have another logical values associated to the $\mathrm{KB}$ that contains the subjects, the metaphor, and the elements of the relation. This corresponds to the HKB. Anthropomorphizations are similar, if not identical, to metaphors.

As in metaphors, subjects will establish a relation between an element of certain phenomenon and will describe it in terms of another phenomenon which corresponds to their domain of action as human beings. Neuroscientists and nowadays physicists enthused with the notion that the brain is the new frontier of scientific knowledge claim that "brains think", which of course, is not the case. A man/woman thinks. No one has been able to isolate a living brain and certainly less to find the attributes of the whole thinking person in this impossible to isolate brain.
This is an instance of the mereological fallacy that reduces the whole to a part (Bennett and Hacker, 2003), yet obliterating the whole and its projection to the part, as well as the wholeness of the part. So this metaphor is about self-reference. It is centered in the thinking person with its embodied thinking and thought body which cannot be separated (Rapoport, 2011b, c), as is the case of the subject that establishes a metaphor, and by establishing the metaphor, the elements of it have been transferred to the human existence, and in particular, its truth value assessment.

The metaphor is not independent of self-reference which is prior to it, be that of the single subject and his fellows that find value in the metaphor. So is the case of scientists that will metaphorize a certain scientific domain as if it would be universal. The latter will call it with the name that reveals its self-referential origin: the Anthropic Principle. (Another such metaphor is 'natural selection', where the self is the Universe, in an undetermined claimedto-be-biological sense). Yet, to transfer this to claim that the state of the universe is determined by a certain metaphor, say a fine tuning of universal constants so that the Universe and we exist simultaneously because we pose the metaphor in the first place, is a somewhat contorted and reductive way of expressing nothing more than a HKB in which the commonness of the metaphor is found valid, yet which requires in the first place the universality of the family of KBs that make the HKB and the inter-relations, and the metacognitive action of metaphorizing even prior yet not independent of the others.

While this certainly establishes a relation between Microcosmos and Macrocosmos and all the in-betweens, yet not of the trivial embeddings of the Matrushkas kind but with self-reenterings of an HKB as in Fig. no. 4, other different 'fine tunings' might be also the case for those who cannot find any meaning to the metaphor, just because their language is not the same as ours, and thus communication if not in terms of universals, is not possible.

These universals return us again thus to the HKB. The 'fine tunings' stand for the universals of some of the elements of the HKB, but seems impossible to state that they 
are valid for all elements of the heterarchy. This would require the claimants to be in a state of identity transparency with the whole heterarchy of elements of the HKB, and this is impossible to verify to be the case. Being the latter the case then we are left with having to establish back the KBL and its ontoepistemology, as a first approximation, or still to take a $\mathrm{KB}$ containing an undeterminable number of related KBs, to elucidate the world in which content and context are unified. This is already our human condition and the condition of all systems.

Thus, the 'universals' cannot be other than context dependent, and this is what has been lately been discovered. Physical (deemed) constants may turn to be context dependent (Webb 1999, 2010; Brax, 2010). To conclude, the HKB which we have found to be the case of the GC, and yet is the case of all systems since this is what is the form of the world, systems are inter-related in heterarchies with reentrances of all elements of them and other selves and the whole reentering in the elements as well, sustains a world in which for a certain subject of the heterarchy, the world seems to be with an outside determined by this subject, and yet reentrance is maintained to be the case. Consistently with this, say other inhabitants living in another area of the Universe, will find themselves another fine tuning to their self-centeredness, and could verify instead another context dependence in their own neighborhood. For an understanding of anthropomorphisms in terms of the differential ontology and epistemology based in the category of distinction (SpencerBrown, 2010), we refer to (Johansen, 2008).

The detour above showed us that the metacognition implicit in the polytopological character of the GC, shows a very rich phenomenology of contextualization, which is the case of the world at large and of all systems. It is through that rich contextualization that only two amino acids as relators of this process of embryological differentiation that appeared from the selfreferential logophysics, in joint action with the environment acting through the remaining amino acids. Evolution, better said unfolding, with regards to the GC, appears as driven by the interaction with the remaining triplets and of the environment at large, in a process of contextualization, not as dual two states as in (Gordon, 2002; Rapoport, 2011c).

Also the KBL provides for the robustness to environmental action; it further generates a representation for consciousness and its fusion to the physical world (Rapoport 2011a, b).

It has been argued in (Rapoport 2009, $2011 \mathrm{~b}, \mathrm{c}$ ) and in this article -see as well (Rosen,2008) -that the $\mathrm{KB}$ has an associated boson spin-1 field associated to a light torsion field, and that is can be seen as a quantum surface, as also we argued to be the case of the interior of the membrane (Rapoport, to appear).

We have reencountered this topology in the GC and discussed the derivation of NUCRS in terms of two KBs, and seen that space appears to be a materialization of the time depth variable, which is essential to hemilateral synchronization of the body (Rapoport 2010b, 2011b). Thus, the double 3D structure of physics, one such space being the spatial coordinates and the other one being the momentum coordinates, has been generated. Since mass is the parameter for momentum space in NUCRS, this has shown that the depth variable that in and through time embodies the bistable perception of the $\mathrm{KB}$ and generates the double $3 \mathrm{D}$, we see that time materializes as space.

This is another materialization of the imaginary (time/depth, as imaginary numbers) as reality (and real numbers). Indeed, we have shown (Rapoport, 2011c) that embryological development of a bauplan, is such materialization!

The dualistic origin of NUCRS which requires a duplication of $3 \mathrm{D}$, was found in the duplication of the $\mathrm{KB}$ to obtain a $\mathrm{HKB}$ which is used to represent the $\mathrm{DH}$, and NUCRS, transforming time -as an operator on a $2 \mathrm{D}$ space- into double $3 \mathrm{D}$-space, as appears in NUCRS, one for the physical space itself, the other for charge. Similarly to the fact that Boolean logic, and dualism at large, is far from having an independent ontological basis other than the KBL (Rapoport 2011a, b) it appears that the $\mathrm{DH}$ has the same status.

We have proposed that this doubling of dimension 3 produced by a superposition of two KBs and further suggested in (Rapoport, 2011c) that it lies at the basis of 
the generation of bauplans, either physical, chemical, biological, etc., in which the imaginary $3 \mathrm{D}$ is related to the time waves in toto generation of bauplans, as a o-time physical-algorithmic causality surmised by Johansen (2008). Its manifestation in the real physical $3 \mathrm{D}$ space, is a world in which the rigid cathegorization that separates Outside and Inside is no longer the case, as shown in the blastopore invagination and in embryological differentiation (Rapoport, 2011c). In the case of the blastopore invagination, we saw that it occurs mediated by pairs of KBs as the ovum's eversion takes place, as if the ovum would be thus creating for itself the representation not just of duality, but of the mutual interpenetration of the imaginary and real $3 \mathrm{D}$ spaces. This is more than a plausible observation, but the actualization of the surmountal of the Cartesian Cut through the unfolding of what will become a full developed embryo.

As shown in NUCRS this duplication produces the most efficient way for physical symmetries coding, and shows up in the rotational generation of the natural numbers from a primeval matrix which allows to identify the primes completely (Johansen, 2011).

So the purported dualism that stands at the basis of NUCRS in (Hill and Rowlands, 2008; Rowlands, 2008) and reappears in physics as momentum and locus representation in space is the result of a nondual logic. Thus, the thesis that NUCRS and physics as derived from it results from a general principle of dualism has been proven not to be the case.

Remarkably, the KB structure of the periodic table of elements, in which each atom and stable nuclide of the table appears, as if existing twice in each side of the onesided topology, has prompted the interpretation that there are atoms of atoms and of antimatter (Boeyens, 2005). Very perspicaciously, this author has seen that there is a continuity between these two kinds of atoms, which in fact can be transformed one into the other, by simply moving inside-out or outside-inwards in the $\mathrm{KB}$ representation of the periodic table. Thus, as in our work, Boeyens was lead to postulate that the $\mathrm{KB}$ is a quantum interface (Boeyens, 2010), and still a projective geometry is the case of the
4D universe. We shall return to this issue elsewhere.

The group symmetry structures -in particular 5 and 8 fold- that appear in the GC (Hill and Rowlands, 2008) -and in its NUCRS translation, showup in evolution (we prefer to name it unfoldment) without selection (Lima de Faria, 1988,1995), and their inter-relation with asymmetries-or still the KB "synsymmetries", fusion of symmetries and asymmetries (Rosen, 2008)as first proposed by Pierre Curie to express the fusion of form and function logophysically the $\mathrm{KB}$ - which was further linked to the structure of the Mendeleev table (through the role atomic weights of atoms in the regularities of biological systems and functionalities (Lima de Faria, 1995), which we know to be the KB (Boeyens, 2005). We recall that its bearing to the generation of the prime numbers from a rotational initial 8 by 8 matrix is crucial (Johansen, 2011).

We may thus propose the novel idea that the periodicities of the taxonomy and functionality of biosystems, as an heterarchy that includes the symmetries of physics, from the physical plenum to the atoms and molecules up to the cosmological scales, must reflect the HKBL of both the GC and the Mendeleev table, incorporating thus the heterarchical fusion of the selfdetermination of biological structures vis-àvis the environment, which far of being 'noise' -which has for geometrical counterpart torsion fields -, it partakes in their constitutional self-referential already present at the diverse physical levels.

In this bauplan for evolution, the Fibonacci sequence of 5 -fold symmetry is to play a major role (Rapoport, 2011c). Johansen has characterized the 5-fold symmetry as the most fundamental algorithm (Johansen, 2008), and in fact the photon as a process has the same kind of self-reference that the Fibonacci sequence (Rapoport 2009, 2011a, b).

Yet inasmuch the atomic periodicities are generated by a standing time wave (Boeyens, 2005) manifesting the Time operator of the KBL (Rapoport 2011a, b, c), we conjecture that this is also the case for biosystems.

Amazingly, such a bauplan for evolution would incorporate already the 
environment, an idea that is reaffirmed by the novel discovery that the actual value of the atomic weights (particularly hydrogen, oxygen and carbon, which could change according to the contexts that produce the different DNA geometries) is not universal but context dependent (Webb 1999, 2010; Brax, 2010; Wieser and Coplen, 2010). This may explain the contextuality of biodiversity and the biological periodicities unveiled by Vavilov (Krassilov, 1989); this author also claimed that species were knots in the evolutionary chain, and thus that the chains themselves would be knots in the higher ecological system evolution:

We find in this idea the HKB heterarchy of evolution, a transformation of

\section{Acknowledgements}

My gratitude to Dr. Sultan Tarlacı, Editor for JNQ, for his kind interest on my work and the invitation together with the Guest Editor, Dr. Tidjani Négadi, to contribute to this special issue. This work was presented as a lecture to the special session "Physics and Logic of Anticipation in Biosystems", CASYS' 11, Conference on Computing Anticipatory Systems, August 2011, by its organizers, Dr Salvatore Santoli, Dr A Grapone, to whom we express our gratitude. To Dr Istvan Diénes for a crucial reference, to Prof $\mathrm{S}$. Johansen for encouragement and to Drs Vanessa Hill \& Peter Rowlands for allowing me to reproduce Fig. 4. To my wife Sonia and my children, Tania and Tsafrir, who gave me all that science and philosophy cannot provide for. context into content into context, which is the onset of the transformations between form and function and their fusion in a HKB heterarchy (Rapoport, 2010 a, b), which is also crucial to consciousness and life (Rapoport 2011a, b, c).

This is conceptually far from Darwinian selection; neither it is the lineartime evolution of creationists, due to the $\mathrm{KB}$ Being, a never ending ever starting selfreference, also present in the NUCRS (which due to its rewrite system appears very much suited to do the job of expressing the biological periodicities), which has now found a logophysical self-referential basis that surmounts the Cartesian Cut.

\section{References}

Annett M. Left, right, hand, and brain. Lawrence Erlbaum, New Jersey, 1985.

Ahmed N and Rao K. Orthogonal Transforms for Digital Signal Processing. Springer, New York, 1975.

Benham C. Unwinding the Double Helix: Using Differential Mechanics to Probe Conformational Changes in DNA.

Calculating the Secrets of Life: Applications of the Mathematical Sciences in Molecular Biology. Lander EC and Waterman MS (eds.) Natl. Acad. Press, Washington,1995.

Bennet M and Hacker P. Philosophical Foundations of Neuroscience. Wiley-Blackwell, London, 2003.

Boeyens, J C. New Theories for Chemistry. Elsevier, Amsterdam, 2005.

Boeyens, J C . Chemical Cosmology. Springer, Berlin, 2010.

Bonchev D, Rouvray DH. Chemical Topology. Gordon \& Breach, London, 2000.

Brax P. Tuning the Mass of Chameleon Fields in Casimir Force Experiments. Phys Rev Lett 2010, 104: 241101.

Brecher J, Van Holde KE and Ahern KG. Biochemistry. Benjamin Cumming, 1999.

Brecher J. Graphical Representation of Stereochemical Configuration. Pure NS Applied Chemistry 2006; 78 ( 10): 1897-1970.

Burdick D. Circular DNA: Double Helix or Moebius strip? Naturwissenchaften 1971 57: 245.

Carlson G. Topology and data. Bull AMS 2009; 46 (2): 255308.

Chang C W, Liu M, Nam S, Zhang S, Liu Y, Bartal G, Zhang X. Optical Moebius Symmetry in Metamaterials 2010; Dec 3;105(23).

Daily $\mathrm{M}$ and Gray J. Allosteric Communication Occus via Networks of Tertiary and Quaternary Motions in Proteins. PLoS Computational Biology 2009; 5 (2): e1000293.

Flapan E. When topology meets chemistry. Cambridge Univ Press, Cambridge, 2000.

Gazale M J. Gnomon: From pharaons to fractals. Princeton Univ. Press, New Jersey, 1999. 
Giuliani A, Di Paola L, Setola R. Proteins as Networks: A Mesoscopic Approach Using Haemoglobin Molecule as Case Study. Current Proteomics 2009; 6: $235-245$.

Goriely A and Tabor M. Nonlinear dynamics of filaments II: Nonlinear analysis. Physica D 1997; $10: 20-44$.

Goriely A. Integrability and Nonintegrability of ordinary differential equations. World Scientific, Singapore, 2001.

Han D, Pal S, Liu Y and Yan H. Folding and cutting DNA into reconfigurable topological nanostructures. Nat Nanotech 2010; 5(10): 712717.

Hill V,Rowlands P. Nature's Code, in Computing Anticipatory Systems. In: CASYS'07-Eighth International Conference; Dubois D M (editor) American Institute of Physics Conference Proceedings. Vol. 1051, pp. 117-126, Springer, Berlin, 2008.

Ishizaki T. Leucine aminopeptidase during meiotic development. Euro J Biochem 2022; 269(3):82632.

Johansen S. Outline of Differential Epistemology. English translation of the original Norwegian, Grunnriss av en differensiell epistemologi. $\left(2^{\text {nd }}\right.$ ed.). Oslo, Abstrakt, 2008.

Johansen S. Complete Exposition of Non-Primes Generated from a Geometric Revolving Approach by $8 \times 8$ Sets of Related Series, and thereby ad negativo Exposition of a Systematic Pattern for the Totality of Prime Numbers. Journal of Dynamical Systems and Geometric Theories 2011; 8 (2): 101171.

Kauffman L. De Morgan algebras, completeness and recursion. In: Proceedings VIIITh International Symposium in Multiple Valued Logics (1978). IEEE Computer Society Press 1978: 82-86.

Kauffman L. Imaginary values in mathematical logic. Proceedings of the Seventeenth International Conference onMultiple Valued Logic, May 26-28 (1987), Boston MA, IEEE Computer Society Press 1987: 282-289.

Krassilov V. Vavilov's species concept and evolution of variation. Evolutionary Theory 1989; 9: 37-44.

Koshland D. Application of a theory of enzyme specifity to protein synthesis. Proc Nat Acad of Sci 1958; 44 (2): 98-104

Kurosawa, Y. \& Okazaki, R. Mechanism of DNA chain growth: XIII. Evidence for discontinuous replication of both strands of P2 phage DNA. J Mol Biology 1975; (2):229-241.

Li BS, Gu LJ, Luo CY et al., The downregulation of asparagine synthetase expression can increase the sensitivity of cells resistant to L-asparaginase. Leukemia 2006; 20: 2199-2201.

Lima de Faría A. Evolution without Selection: Form and Function by Autoevolution. Elsevier, Amsterdam, 1988; ibid. The atomic basis of biological symmetry and periodicity, Biosystems 1997; 43 (2):115-135.

Lima de Faría A. Biological periodicity, Greenwich, JAI Press,USA, 1995.

Petoukhov S, He M. Symmetrical Analysis Techniques for Genetic Systems and Bioinformatics. IGI Global, New York, 2010.
Petoukhov S, He M. Genetic code, Hamming distance and stochastic matrices. Bull Math Biol 2004; 66: 1405-1421.

Rapoport DL. Cartan-Weyl Dirac and Laplacian operators, Brownian motions, The quantum potential and Scalar Curvature, Dirac-Hestenes equations and supersymmetric systems. Found Phys 2005 ; 35 ( 8): 1383-1431.

Rapoport DL. Torsion Fields, the Extended Photon, Quantum Jumps, the Eikonal Equations, the Twistor Geometry of Cognitive Space and the Laws of Thought. In: Ether, Spacetime and Cosmology vol. 3: Physical Vacuum, Relativity and Quantum Mechanics. Duffy M and Levy J (editors), 389457, Apeiron Press, Quebec, Canada.

Rapoport DL. Torsion, propagating singularities, nilpotence, quantum jumps and the eikonal equations. In: Computing Anticipatory Systems, Proceedings CASYS'09, Daniel M. Dubois (ed.), AIP Conf.Series 1303. Springer, Berlin, 2010a.

Rapoport DL. Self-reference, the Moebius and Klein bottle surfaces,multivalued logic and cognition, International Journal of Computing Anticipatory Systems 2010b; 23: 103-113.

Rapoport DL. Surmounting the Cartesian with Philosophy, Physics, Logic, Cybernetics and Geometry: Self-reference, torsion, the Klein bottle, the time operator, multivalued logics and quantum mechanics, Found Phys 2011 a; 41 (1): 33-76.

Rapoport DL. Surmounting the Cartesian Cut Further: Torsion Fields, the Extended Photon, Quantum Jumps, The Klein Bottle, Multivalued Logic, the Time Operator, Chronomes, Perception, Semiosis, Neurology and Cognition. In: Focus in Quantum Mechanics, D. Hathaway and E. Randolph (eds), Nova Science, NY, 2011b.

Rapoport DL. On the Fusion of Physics and Klein Bottle Logic in Embryogenesis, Cell Biology and Evolution, 2011c, submitted to JNQ.

Rapoport DL. Klein Bottle Logophysics: Cell Biology, Chemistry and Body Integration. To appear.

Rich A. The nucleic acids. In: DNA, The Double Helix: Perspective and Prospective at Forty Years. New York Academy of Sciences, Chambers D (editor); New York, 1995.

Rosen SM. The Self-evolving Cosmos: A Phenomenological Approach to Nature's Unity-indiversity. World Sientific, Singapore, 2008.

Rowlands P. From Zero to Infinity. World Scientific, Singapore, 2008.

Herges R. Topology in Chemistry: Designing Moebius Molecule . Chemical Reviews 2006; 106: 482.

Savir Y, Tlusty T. Conformational Proofreading: The Impact of Conformational Changes on the Specificity of Molecular Recognition. PLos One 2007; 5: e468.

Schill G, Zurcher C . Naturwissenschaften 1971; 58: 40-45.

Seeman N C. DNA Nanotechnology: Novel DNA Constructions. Annu Rev Biophys Biomol Struct 1998; $27: 225-48$.

Sokolov I. Topological Methods in Stereochemistry. Russ Chem Rev 1973; 42 (6).

Sopanen T, Uuskallio M, Nyman S, Mikola J. Characteristics and Development of Leucine Transport Activity in the Scutellum of Germinating 
Barley Grain Plant Physiology 1980; 65 (2): 249253

Spencer-Brown G. Laws of Form, Bohmeier Verlag, Leipzig, 2010.

Stern A. Quantum Theoretic Machines. Elsevier, Amsterdam, 2001.

Uversky VN. Natively unfolded proteins: a point where biology waits for physics. Protein Sci 2002; 11, 739756.

Varela F. Principles of Biological Autonomy. NorthHolland, New York, 1979 .

Waterman, M. S. (Ed.). Mathematical methods for DNA sequences, CRC Press, 1999.

Webb JK, King JA, Murphy MT, Flambaum VV, Carswell RF, Bainbridge MB. Indications of a spatial variation of the fine structure constant. Phys Rev Lett 2010; 82:884.

Werner G. The Topology of the Body Representation in the Somatic Afferent Pathway. In: The Neurosciences Second Study Program, pp. 605-
617, Schmitt,F.O. (ed.),Rockefeller University, 1968.

Wieser $\mathrm{M}$ and Coplen T. Atomic weights of the elements 2009 (IUPAC Technical Report). Pure Appl Chem 2011, Vol. 83, No. 2: 359-396

Ycas M. The biological code. North-Holland, Amsterdam, 1969.

Yoshikazu K, Okazaki R. Mechanism of DNA chain growth: XIII. Evidence for discontinuous replication of both strands of $\mathrm{P} 2$ phage DNA. J Mol Biology 1975; 94(2): 229-241

Yudelvich A, Ginsberg A, Hurwitz J. Discontinuous synthesis of DNA during replication. Proc Natl Acad Sci USA 1968; 61, 1129.

Zheng J, Birktoft JJ,Chen Y, Wang T, Sha R, Constantinou P,Ginell G,Mao G, Seeman NC. From Molecular to Macroscopic via the Rational Design of a Self-Assembled 3D DNA Crystal. Nature, 2009, September 3; 461(7260): 74-77. 